\title{
An essay on diabetes from a biochemist
}

\begin{abstract}
Aizawa $\mathrm{H}^{*}$
Aizawa Science Museum, Kasukabe, Saitama, Japan

Since plants started to produce glucose as a stock of sun energy by photosynthesis, majority of the living things on the earth have used glucose as an energy source. Glucose is an essential unit component of polysaccharides such as glycogen. We also use glucose as a material for glycosylation of polypeptides and lipids to produce glycoproteins and glycolipids, respectively.
\end{abstract}

Glucose is one of reducing sugars, which take three types of chemical structure, that is, alpha, beta, and linear types. The latest one has aldehyde group, which could react with an amine group of polypeptides by a nucleophilic reaction. The resultant Schiff's base is irreversibly converted to a chemically stable keto-amine derivative. This chemical modification by glucose is called glycation. For example, haemoglobin is glycated at its amino-terminal amine group by blood glucose to some extent, which is an important index for diagnosis of diabetes mellitus.

Glycation causes various serious symptoms such as vascular disorders, renal dysfunction, and vision loss. Blood glucose slowly and continuously glycates surface proteins of blood vessel epithelial cells by chance. Glycation may modulate function of surface proteins to degrade qualities. Moreover, glycated surface proteins are recognized as alien antigens by immune system. Consequently, the epithelial cells, whose surface proteins are glycated, are attacked by neutrophils using oxidative weapons and engulphed by macrophages. In case of chronic high blood sugar patients, activated immune response may cause inflammation, which further damages surrounding tissues by oxidative stress. In a rare case, oxidative modification of membrane receptors constitutively accelerates cell growth and proliferation, which might cause cancer development. Taken all together, suppression of the glycation could improve quality of life of diabetic patients.

Modern treatments for diabetes are mainly focused on the way how to decrease blood glucose level to normal one. Injection of insulin in vein could be effective to increase the uptake of blood glucose by cells such as adipocyte, muscle, and liver. Also, drugs that increase sensitivity of insulin receptor could reduce the blood glucose level. It is also effective to reduce reabsorption of glucose by drugs which inhibit sodium glucose cotransporter in renal tubular epithelial cells.

Copyright: (C2018 Aizawa H. This is an open-access article distributed under the terms of the Creative Commons Attribution License, which permits unrestricted use, distribution, and reproduction in any medium, provided the original author and source are credited.
Type-I diabetes patients have defect in insulin release from islet beta cells. Both auto immune disease against beta cells and chronicle hyperglycemia are known to develop type I diabetes. Hyperglycemia increases glucose concentration in islet beta cells, which lose homeostasis of redox system by relative short of oxygen to induce apoptosis. In type-I diabetes patients, it is effective to stimulate insulin release from islet beta cells pharmacologically or to apply insulin directly in vein after each meal. It is also effective to increase the sensitivity of target cells such as adipocytes, muscles, and liver cells to insulin.

In type-II diabetes patients, adipocytes reach to their maximum limit of lipid storage and thus their sensitivity to insulin stimulation should be suppressed by negative feedback. In such a case, an application of large dose of insulin does not decrease blood glucose level efficiently. Pharmacological inhibition of the negative feedback reduces blood glucose level effectively, while chronic administration might put a heavy burden on adipocytes in patients. On the other hand, inhibition of tubular reabsorption of glucose could be effective for longterm treatment to decrease blood glucose level, while this treatment might cause hypoglycemia or bacterial infection in the excretory organ as side effects.

For radical cure, however, type-II diabetes patients need to recover sensitivity to insulin by burning fats in their adipocytes. Dietary and exercise treatments are most effective. Exercise induces secretion of stress hormones such as epinephrine and norepinephrine, which induce degradation of fats to produce energy. Once adipocytes could have room for storage of fats, they could respond to insulin to maintain blood glucose at normal level.

Recently, the number of patients of diabetes keeps on growing to around seven percent of total population of human beings. Diabetes is a new disease derived from civilized society with enough food supply, and thus it is quite important for modern society to develop preventive medicine for diabetes.

I hope that clinical physicians and research scientists collaborate all over the world to accelerate education, research, and development of prevention and treatment of diabetes mellitus for benefit of human beings.
*Correspondence to: Hiroyuki Aizawa, Aizawa Science Museum, 1-10-13 Yutaka-cho, Kasukabe, Saitama 3440066, Japan, Tel: +81-48-754-9880; Fax: +81-48-754-9880, E-mail: aizawa@rr.iij4u.or.jp

Received: August 07, 2018; Accepted: August 20, 2018; Published: August 22, 2018 